Ernst Kurths Grundlagen des linearen Kontrapunkts 
Luitgard Schader

\section{Ernst Kurths \\ Grundlagen \\ des linearen \\ Kontrapunkts}

Ursprung und Wirkung

eines musikpsychologischen Standardwerkes

Verlag J. B. Metzler

Stuttgart · Weimar 


\section{30}

Die Deutsche Bibliothek - CIP-Einheitsaufnahme

\section{Schader, Luitgard:}

Ernst Kurths Grundlagen des linearen Kontrapunkts : Ursprung und Wirkung eines musikpsychologischen Standardwerkes/ Luitgard Schader. - Stuttgart ; Weimar : Metzler, 2001

(M-\&-P-Schriftenreihe für Wissenschaft und Forschung) ISBN 978-3-476-45260-3 ISBN 978-3-476-02780-1 (eBook) DOI 10.1007/978-3-476-02780-1

Dieses Werk einschließlich aller seiner Teile ist urheberrechtlich geschützt. Jede Verwertung außerhalb der engen Grenzen des Urheberrechtsgesetzes ist ohne Zustimmung des Verlages unzulässig und strafbar. Das gilt insbesondere für Vervielfältigungen, Übersetzungen, Mikroverfilmungen und die Einspeicherung und Verarbeitung in elektronischen Systemen.

M \& P Schriftenreihe für Wissenschaft und Forschung

(C) 2001 Springer-Verlag GmbH Deutschland

Ursprünglich erschienen bei J.B.Metzlersche Verlagsbuchhandlung und Carl Ernst Poeschel Verlag GmbH in Stuttgart 2001 


\section{Inhalt}

Dank VII

Zur Textgestaltung ................................................................................................

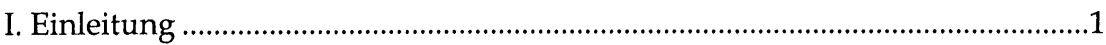

II. Ernst Kurth

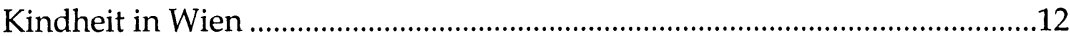

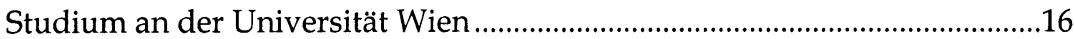

Das Jahr in Wickersdorf ...............................................................................24

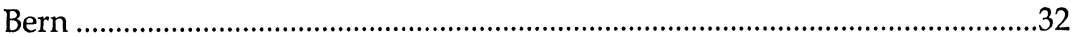

III. Ernst Kurth und die Gestaltpsychologie

Die Ursprünge der modernen Gestaltpsychologie..............................................55

Die Anfänge der Berliner Schule für Gestaltpsychologie...................................59

Ernst Kurth und Max Wertheimer....................................................................66

IV. Grundlagen des linearen Kontrapunkts

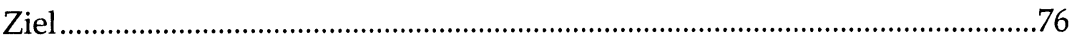

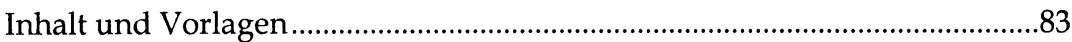

V. Reaktionen auf den Linearen Kontrapunkt .......................................................109

VI. Auseinandersetzung mit der Theorie durch die Musikwissenschaft

Die Interpretation Hugo Riemanns ..................................................................122

Die Rezeption im deutschsprachigen Raum zwischen den Kriegen ............129

Der Lineare Kontrapunkt nach dem Zweiten Weltkrieg.................................168

VII. Analyse-Kriterien für »lineare« Kompositionen der 1910er und 20er Jahre

Kurths Kriterien eines polyphonen Stils der Bach-Zeit .................................187

Übertragbare Analyse-Kriterien für lineare Kompositionen ..........................196

VIII. August Halm (1869-1929) …………………...................................................199

IX. Komponisten der Berliner Gruppe..............................................................205

Artur Schnabel (1882-1951) .........................................................................206

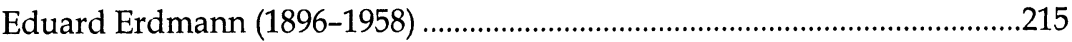

Heinz Tiessen (1887-1971) ...........................................................................224

Ernst Krenek (1900-1991) …………………………....................................233 
X. Paul Hindemith (1895-1963)

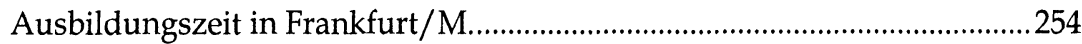

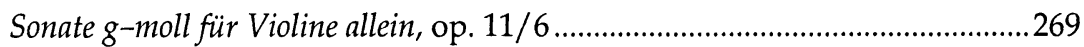

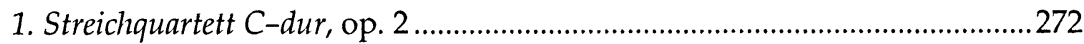

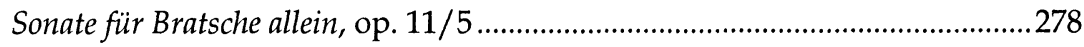

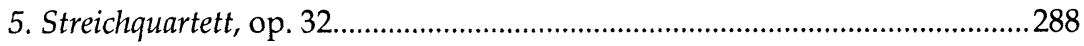

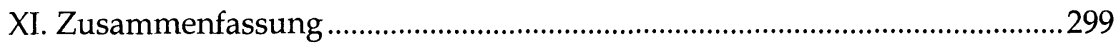

Anhang

Der Briefwechsel zwischen Ernst Kurth und Paul Bekker...............................307

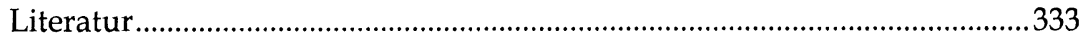

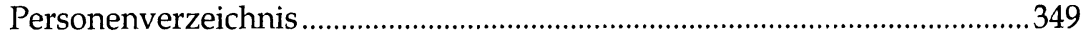




\section{Dank}

Die Beschäftigung mit Ernst Kurths Schrift Grundlagen des linearen Kontrapunkts führt weit über die Interpretation publizierter Quellen hinaus. Denn Kurth lebte zurückgezogen; über die Zeit seiner Kindheit, Jugend und Ausbildung in Wien gab er selbst im Familienkreis nur wenige Auskünfte, und seine Kontakte zu Kollegen im eigenen Fach oder zu Wissenschaftlern der Nachbardisziplinen blieben bisher nahezu unerforscht.

Um das Ziel der vorliegenden Arbeit erreichen zu können, nämlich Ernst Kurths Linearen Kontrapunkt im Umfeld der musikwissenschaftlichen Forschung und der kompositorischen Entwicklung der 1910er und 20er Jahre zu interpretieren, war ich deshalb primär auf Hinweise aus bisher unbekannten Dokumenten Ernst Kurths und auf die Berichte vieler Zeitzeugen angewiesen, die ich in Gesprächen und Briefen erhielt.

Bei meiner langwierigen Suche nach Informationen begegnete mir ein großes Interesse und die ausgesprochen entgegenkommende Hilfsbereitschaft vieler Personen und Institutionen, wofür ich an dieser Stelle allen herzlich danken möchte.

An erster Stelle ist dabei Hans Kurth, Muri bei Bern, zu nennen, der die Entwicklung meiner Arbeit in allen Stadien begleitete. Hans Kurth stellte mir nicht allein die Quellen aus dem Nachlass seines Vaters zur Verfügung, sondern bestätigte einige meiner Vermutungen aus seiner Erinnerung und ergänzte in vielen Gesprächen einzelne Begebenheiten aus der Biographie Ernst Kurths.

Professor Michael Wertheimer, Univerity of Colorado at Boulder, gab mir in Briefen wertvolle Informationen zur Biographie und zur Forschung seines Vaters Max Wertheimer.

Barbara Bekker Rawling erlaubte den Abdruck der Briefe ihres Vaters Paul Bekker an Ernst Kurth.

Professor Kurt von Fischer, zunächst Zürich, jetzt Bern, berichtete in Briefen und Gesprächen von seinen persönlichen Begegnungen mit Ernst Kurth.

Professor Anselm Gerhard, Universität Bern, gab wertvolle Anregungen zur Druckfassung der Arbeit.

Nach der Erkrankung von Professor Helmut Hucke betreute Professor Winfried Kirsch meine Arbeit, auch ihm sei dafür gedankt. 
Mein Dank gilt auch den Mitarbeiterinnen und Mitarbeitern

- des Universitätsarchivs Bern

- des Schiller Nationalmuseums/Deutsches Literaturarchiv, Marbach/N.

- der Wiener Stadt- und Landesbibliothek

- des Archivs der Universität Wien

- der Bayerischen Staatsbibliothek in München

- der Yale Univesity, Irving S. Gilmore Music Library, New Haven

- der Universität Erlangen-Nürnberg, die Teile des Bruno Stäblein Archivs betreuen

für ihre vielen Hinweise auf unveröffentlichte Quellen zu Ernst Kurth.

Friedrichsdorf/Taunus im Januar 2001

Luitgard Schader 


\section{Zur Textgestaltung}

Ernst Kurth veröffentlichte seine Schrift Grundlagen des linearen Kontrapunkts in drei Auflagen, die sich leicht voneinander unterscheiden.

Wenn nicht anders hervorgehoben, bezieht sich die hier vorliegende Arbeit auf einen Nachdruck der dritten Auflage, der 1977 bei Olms in Hildesheim erschien. Diese Quelle überliefert den Text in nahezu vollständiger Fassung und ist darüber hinaus heute allgemein zugänglich. Sie unterscheidet sich von der zweiten Auflage (Hesse, Berlin 1922) durch die Ergänzung des Vorworts zur dritten Auflage (das Vorwort zur zweiten Auflage entfiel) und die Korrektur einiger Druckfehler - so sind beispielsweise die nicht gedruckten letzten vier Worte auf Seite 29 sind in der dritten Auflage ergänzt.

Offensichtliche Druckfehler in der Ausgabe des Linearen Kontrapunkts wurden in der vorliegenden Arbeit stillschweigend korrigiert. Die historische Orthographie der älteren Quellen wurde hingegen in Zitaten grundsätzlich beibehalten. 